\title{
FRECUENCIA DE LA CESÁREA EN ANDALUCÍA. RELACIÓN CON FACTORES SOCIALES, CLÍNICOS Y DE LOS SERVICIOS SANITARIOS (2007-2009)
}

\author{
Soledad Márquez-Calderón (1), Miguel Ruiz-Ramos (2), Sol Juárez (3) y Julián Librero \\ López (4)
}

(1) Secretaría General de Salud Pública y Participación. Consejería de Salud de la Junta de Andalucía.

(2) Servicio de Información y Evaluación. Consejería de Salud de la Junta de Andalucía.

(3) Instituto de Economía, Geografía y Demografía. Consejo Superior de Investigaciones Científicas.

(4) Centro Superior de Investigación en Salud Pública de Valencia.

\section{RESUMEN}

Fundamento: La tendencia creciente y variabilidad geográfica en el uso de la cesárea sugiere la existencia de factores explicativos no clínicos. El objetivo del estudio es analizar el uso de las cesáreas en Andalucía, explorando el papel de variables sociales, clínicas y de los servicios sanitarios.

Métodos: Estudio transversal de todos los nacimientos de Andalucía (2007-2009) utilizando como fuente de información el Movimiento Natural de la Población. La variable dependiente fue la frecuencia de cesáreas y las explicativas se clasificaron en tres grupos: con significado clínico, relacionadas con el funcionamiento de los servicios sanitarios, y con significado social. Se realizó una regresión logística multivariante.

Resultados: El 24,8\% de los 293.558 nacimientos fue por cesárea. En el análisis multivariante se observó que la variable clínica con la mayor odds ratio (OR) fue la existencia de complicaciones $(\mathrm{OR}=19,36)$. En cuanto a las variables relacionadas con los servicios sanitarios hubo un 55\% más cesáreas de lunes a viernes que en fines de semana y la provincia con mayor uso de cesárea fue Cádiz (OR de 1,21 frente a Almería) con una razón de partos en centros públicos frente a privados de 3,7 . La frecuencia de cesáreas fue un $34 \%$ superior en las mujeres con estudios de tercer grado que en las que no tenían estudios.

Conclusiones: La variable con mayor influencia en la práctica de cesárea fue la existencia de complicaciones. La tasa de cesárea estuvo por encima de los estándares aceptados en todas las clases sociales, con mayor incidencia a mayor nivel educativo. Las diferencias interprovinciales reflejan distintos patrones de uso de la medicina privada.

Palabras clave: Cesárea. Calidad de la atención de salud, acceso y evaluación. Desigualdades en la salud.

\section{Correspondencia:}

Soledad Márquez-Calderón

Secretaría General de Salud Pública y Participación

Consejería de Salud de la Junta de Andalucía

Avenida de la Innovación s/n

Edificio Arena 1

41020 Sevilla

soledadm.marquez@juntadeandalucia.es

\section{ABSTRACT}

\section{Caesarean Delivery in Andalusia, Spain. Relationship with Social, Clinical and Health Services Factors (2007-2009)}

Background: Increasing trend and geographical variations in the use of caesarean section suggest the influence of non-clinical factors. The objective was to describe the use of caesarean section in the Andalusian region in Spain by exploring the role of social, clinical, and health services variables.

Methods: A cross-sectional study was carried out using vital statistics. It involves all births occurred in Andalusia during the period of 2007-2009. The dependent variable was the use of caesarean section and the set of covariates were classified into three groups: those with a clinical meaning, those related to the health services organization, and those with a social significance. Multivariate logistic regressions were used.

Results: In the data set of 293,558 births, the prevalence of caesarean delivery was $24.8 \%$. The multivariate analysis highlights the labour complications as the clinical variable with the highest odds ratio $(\mathrm{OR}=19.36)$. Regarding the health services variables, the odds of experiencing a caesarean delivery were $55 \%$ higher on weekdays than on weekends. Cádiz was the province with the highest OR for caesarean section (comparison between Cádiz and Almería: $\mathrm{OR}=1,21$ ) where the ratio between births in public and private hospitals was 3.7. The frequency of caesarean section was $34 \%$ higher in women with third level education than those with no education.

Conclusions: Labour complication is the most influential variable for caesarean section. Caesarean birth rate is above the accepted standards for all social classes and increases with educational level. Inter-provincial differences reflect different patterns with regard to the use of private medicine.

Key words: Cesarean section. Health care quality, access and evaluation. Health social inequalities. 


\section{INTRODUCCIÓN}

El incremento a lo largo del tiempo del uso de la cesárea está descrito desde hace años en muchos países. Un artículo con datos de Estados Unidos constataba que se había pasado de un 5,5\% de nacimientos por cesárea en 1970 a un $22,8 \%$ en 1993 , con una importante variabilidad geográfica y con mayores tasas en los hospitales privados ${ }^{1}$. En España se ha documentado un incremento del porcentaje de nacimientos por cesárea desde un $13,6 \%$ a finales de los años setenta hasta un $25,1 \%$ en 2001-2002, ocurriendo esta tendencia en todos los grupos de edad materna excepto en las mujeres mayores de 40 años ${ }^{2}$.

Esta tendencia en el uso de la cesárea es objeto de preocupación y se considera un hecho no deseable por diversos motivos. En primer lugar, no parece que responda a cambios en factores biológicos o clínicos. Aunque algunos de estos factores pudieran explicar una parte del aumento de las cesáreas (como el aumento de la edad de la mujer en la maternidad o el mayor porcentaje de partos múltiples con fetos de bajo peso, por el uso de la fertilización in vitro) sería una parte pequeña. En segundo lugar, la variabilidad geográfica y entre hospitales, así como la influencia de factores no clínicos -como la titularidad de la clínica donde ocurre el nacimiento, pública o privada- ponen de manifiesto que pueden existir explicaciones relacionadas con la discrecionalidad de la práctica clínica y las propias preferencias de las mujeres ${ }^{3-5}$. Por otra parte, existe una controversia importante en la literatura acerca de si éticamente puede justificarse la realización de cesárea a demanda de la mujer ${ }^{6}$.

Algunos estudios han abordado recientemente el tema del acceso a la cesárea bajo el enfoque de las desigualdades sociales y por etnia $^{7,8}$. En el caso de Brasil, la tasa de nacimientos por cesárea en mujeres primíparas varió desde un $23 \%$ en las de menor nivel educativo a un $69 \%$ en las de mayor nivel, siendo también superior en las mujeres blancas que en las de otros grupos étnicos ${ }^{7}$. En la ciudad de Barcelona el porcentaje de nacimientos por cesárea fue del $23,7 \%$ en mujeres trabajadoras manuales y del 33,1\% en las trabajadoras nomanuales para el período 1994-2003. Esta diferencia por clase social se explicó sobre todo en base al tipo de maternidad, con mayor uso en las clínicas privadas ${ }^{8}$.

Si bien estos estudios ponen de manifiesto un menor uso de la cesárea en los grupos sociales más desfavorecidos, también es cierto que incluso en estos grupos la tasa supera el $15 \%$ recomendado por la Organización Mundial de la Salud ${ }^{9}$. Por este motivo podría considerarse que existe una sobreutilización generalizada de la cesárea que estaría más relacionada con aspectos de la medicalización del nacimiento que con necesidades por motivos clínicos.

En este contexto se plantea el objetivo de analizar el uso de las cesáreas en las mujeres residentes en Andalucía, explorando el papel que juegan el nivel educativo (como aproximación a la clase social), las variables clínicas que podrían justificar este procedimiento y los factores relacionados con el funcionamiento de los servicios sanitarios.

\section{SUJETOS Y MÉTODO}

Se realizó un estudio transversal para el periodo de 2007 al 2009 utilizando los datos de nacimientos procedentes del Movimiento Natural de la Población (MNP) del Instituto Nacional de Estadística y el Instituto de Estadística de Andalucía. El MNP es una fuente de datos exhaustiva, ya que comprende todos los nacimientos que ocurren en España. La información es cumplimentada por los padres en el boletín estadístico de partos. La última versión del boletín (de 2007) incorpora algunas variables nuevas (como el nivel de estudios de los padres) y facilita la comprensión de algunos términos respecto a versiones anteriores (como el cambio de "parto distócico" por "parto con complicaciones"). 
En el análisis realizado en este estudio se incluyeron todos los nacimientos ocurridos en Andalucía de mujeres residentes en esta comunidad. La variable dependiente fue si el nacimiento se realizó mediante cesárea o por vía vaginal. Las variables explicativas se clasificaron en tres grupos en función del objetivo de la investigación. El primero se denominó variables con significado clínico, incluyendo las siguientes: existencia de complicaciones en el nacimiento (se refiere a la codificación como parto complicado o normal en el boletín estadístico de partos); edad de la madre (menores de 20 años, de 20 a 29 , de 30 a 39 e igual o mayor de 40 ); si la mujer era primípara o no; peso del recién nacido (menos de 2.500 gramos, entre 2.500 y 3.999 , e igual o mayor a 4.000); tiempo de gestación (menor de 32 semanas, entre 32 y 36 , de 37 a 42 y mayor de 42); y sexo del recién nacido. El segundo grupo incluyó variables que podrían estar relacionadas con el funcionamiento de los servicios sanitarios: día de la semana en que ocurrió el nacimiento (categorizado en sábado y domingo por un lado, y de lunes a viernes por otro) y la provincia de residencia de la madre (con interés debido a las diferencias provinciales en el uso de la medicina privada versus pública). Por último, en el tercer grupo se incluyeron las variables con significado social: nivel de estudios de la madre ( $\sin$ estudios, primer grado, segundo grado primer ciclo, segundo grado segundo ciclo, y tercer grado); país de origen (con 6 categorías: España, Europa del Este, Magreb, África sin el Magreb, Asía, y Europa del oeste, sur, norte, EEUU y Australia) y si la mujer vivía en pareja o no. Para el nivel de estudios se agregó la información siguiendo las indicaciones de la Sociedad Española de Epidemiología ${ }^{10}$

Con estas variables se construyeron tres modelos multivariantes de regresión logística binaria hacia delante. El primer modelo incluyó las variables con significado clínico, el segundo añadió las variables relacionadas con el funcionamiento de los servicios sani- tarios, y el tercero (modelo principal) añadió a las anteriores las variables con significado social. De esta forma al pasar de un modelo al siguiente podía verse si cambiaba el efecto de las variables de cada grupo. Para cuantificar la importancia de cada una de las variables independientes sobre el uso de cesárea se calculó la odds ratio (OR) y su intervalo de confianza al $95 \%$.

Como es conocido, una de las circunstancias que más influyen en el uso de la cesárea es el tipo de hospital (privado o público). Este dato no consta en el registro de partos del MNP, por lo que no pudo analizarse directamente. Como aproximación se realizó una descripción del porcentaje de partos y de cesáreas según titularidad del hospital en cada una de las provincias andaluzas, basado en la Encuesta de Establecimientos Sanitarios con Régimen de Internado de 2007.

\section{RESULTADOS}

En Andalucía, para el periodo de 2007 a 2009 , de un total de 293.558 nacimientos, 72.802 fueron por cesárea $(24,8 \%)$ (tabla 1$)$.

En el análisis descriptivo de los datos la variable que más relación tuvo con las cesáreas fue la existencia de complicaciones, con 28.572 cesáreas en 35.449 nacimientos complicados (81\%), frente a 44.739 en 255.653 nacimientos no complicados (17\%). La edad de la madre tuvo un comportamiento lineal, con 964 cesáreas en 5.783 nacimientos de mujeres menores de 20 años $(16,7 \%)$ y 10.257 en 33.741 nacimientos de mujeres mayores de 39 años (30,4\%). El porcentaje de cesáreas fue superior en las mujeres primíparas que en el resto, con 42.713 cesáreas en 152.004 nacimientos de primíparas $(28,1 \%)$. El peso al nacer también influyó en el porcentaje de cesáreas, que fue especialmente alto en los recién nacidos de bajo peso, con $10.882(46,6 \%)$ cesáreas en 23.353 nacimientos, seguido de los macrosómicos. 
Durante los fines de semana el porcentaje de cesáreas fue inferior al resto de los días de la semana, con $15.220(20,6 \%)$ cesáreas en 73.887 nacimientos ocurridos en sábados o domingos, frente a $27,4 \%$ de lunes a viernes. Por provincia de residencia de la madre destacaron Cádiz y Málaga en un extremo, con 29.401(29,5\%) cesáreas en 99.667 nacimientos ocurridos en estas dos provincias, y Huelva y Jaén en el otro.
Se puso de manifiesto un gradiente en el uso de la cesárea según nivel de estudios de la madre, en el sentido de mayor uso a medida que aumentaban los años de educación, oscilando entre $11.898(22,6 \%)$ cesáreas en 52.644 nacimientos en las mujeres sin estudios y $19.533(27,7 \%)$ cesáreas en 70.515 nacimientos en las que tenían estudios de tercer grado. En 248.109 nacimientos de mujeres que convivían en pareja se dieron

\section{Tabla 1}

Variables asociadas al uso de la cesárea en Andalucía desde 2007 al 2009

\begin{tabular}{|c|c|c|c|}
\hline Variables & $\begin{array}{l}\text { Número de } \\
\text { partos }\end{array}$ & $\begin{array}{c}\% \text { del total de } \\
\text { partos }\end{array}$ & \% de cesáreas \\
\hline \multicolumn{4}{|c|}{ Complicaciones en el nacimiento } \\
\hline Sin complicaciones & 255.653 & 87,1 & 17,5 \\
\hline Con complicaciones & 35.449 & 12,1 & 80,6 \\
\hline Perdidos & 2.456 & 0,8 & \\
\hline \multicolumn{4}{|l|}{ Edad de la madre } \\
\hline$<20$ años & 5.783 & 2,0 & 16,7 \\
\hline 20-29 años & 69.307 & 23,6 & 22,3 \\
\hline 30-39 años & 179.335 & 61,1 & 26,4 \\
\hline$<=40$ años & 33.741 & 11,5 & 30,4 \\
\hline Perdidos & 5.392 & 1,8 & \\
\hline \multicolumn{4}{|l|}{ Primiparas } \\
\hline $\mathrm{Si}$ & 152.004 & 51,8 & 28,1 \\
\hline No & 141.554 & 48,2 & 23,1 \\
\hline \multicolumn{4}{|l|}{ Peso al nacer } \\
\hline$<2500$ gramos & 23.353 & 8,0 & 46,6 \\
\hline $2500-3999$ gramos & 243.315 & 82,9 & 23,2 \\
\hline$>=4000$ gramos & 17.873 & 6,1 & 33,6 \\
\hline Perdidos & 9.017 & 3,1 & \\
\hline \multicolumn{4}{|c|}{ Sexo del recien nacido } \\
\hline Niño & 151.632 & 51,7 & 25,5 \\
\hline Niña & 141.926 & 48,3 & 24,0 \\
\hline \multicolumn{4}{|l|}{ Tiempo de gestación } \\
\hline$<32$ semanas & 2.303 & 0,8 & 54,4 \\
\hline $32-36$ semanas & 17.271 & 5,9 & 43,5 \\
\hline $37-42$ semanas & 208.499 & 71,0 & 23,7 \\
\hline$>42$ semanas & 256 & 0,1 & 25,8 \\
\hline Perdidos & 65.229 & 22,2 & \\
\hline \multicolumn{4}{|l|}{ Día de la semana } \\
\hline Sábado-domingo & 73.887 & 25,2 & 20,6 \\
\hline Lunes-viernes & 219.671 & 74,8 & 27,4 \\
\hline
\end{tabular}


Tabla 1

Variables asociadas al uso de la cesárea en Andalucía desde 2007 al 2009 (cont.)

\begin{tabular}{|c|c|c|c|}
\hline \multicolumn{4}{|l|}{ Provincia residencia de la madre } \\
\hline Almeria & 26.210 & 8,9 & 25,3 \\
\hline Cádiz & 44.279 & 15,1 & 29,5 \\
\hline Córdoba & 25.548 & 8,7 & 25,9 \\
\hline Granada & 31.047 & 10,6 & 21,4 \\
\hline Huelva & 17.839 & 6,1 & 20,3 \\
\hline Jaén & 20.483 & 7,0 & 21,1 \\
\hline Málaga & 55.388 & 18,9 & 29,4 \\
\hline Sevilla & 72.764 & 24,8 & 24,9 \\
\hline \multicolumn{4}{|l|}{ Nivel de estudios de la madre } \\
\hline Sin estudios & 52.644 & 17,9 & 22,6 \\
\hline Primer grado & 84.265 & 28,7 & 25,1 \\
\hline Segundo grado, 1 ciclo & 51.416 & 17,5 & 26,6 \\
\hline Segundo grado, 2 ciclo & 23.825 & 8,1 & 27,1 \\
\hline Tercer grado & 70.515 & 24,0 & 27,7 \\
\hline Perdidos & 10.893 & 2,7 & \\
\hline \multicolumn{4}{|l|}{ Vive en pareja } \\
\hline $\mathrm{Si}$ & 248.109 & 84,5 & 26,1 \\
\hline No & 45.449 & 15,5 & 23,1 \\
\hline \multicolumn{4}{|l|}{ País de origen de la madre } \\
\hline España & 258.439 & 88,0 & 25,8 \\
\hline $\begin{array}{l}\text { Europa oeste-sur-norte, EEUU y } \\
\text { Australia }\end{array}$ & 4.422 & 1,5 & 24,9 \\
\hline Europa del Este & 8.025 & 2,7 & 18,8 \\
\hline Magreb & 9.342 & 3,2 & 25,0 \\
\hline África, excepto Magreb & 1.192 & 0,4 & 33,8 \\
\hline América del sur & 10.167 & 3,5 & 27,2 \\
\hline Asía & 1.920 & 0,7 & 23,0 \\
\hline Perdidos & 51 & 0,0 & \\
\hline Total & 293.558 & 100,0 & 24,8 \\
\hline
\end{tabular}

$64.756(26,1 \%)$ cesáreas, porcentaje superior que en las mujeres que vivían solas $(23,1 \%)$. En cuanto al país de origen de la madre el número de cesáreas en las mujeres africanas fue $403(33,8 \%)$ de un total de 1.192 nacimientos, siendo las de Europa del Este las que tuvieron menos: 1.509 (18,8\%) nacimientos por cesárea de un total de 8.025 (tabla 1).

En los sucesivos modelos de regresión logística multivariante que se utilizaron (tabla 2) se observó que la variable existencia de complicaciones siguió siendo la de mayor influencia sobre la realización de cesárea (OR entre 18,71 y 19,36 según el modelo), manteniéndose esta influencia cuando se introdujeron las variables relacionadas con el funcionamiento de los servicios sanitarios y las variables sociales (OR de 19,36, tras ajustar por todas las variables). De igual manera se mantuvo prácticamente sin cambios el gradiente de cesáreas según edad de la madre (con una OR ajustada por todas las demás variables de 2,43 al comparar las mayores de 39 años con las menores de 20), primiparidad (OR ajustada de 0,78 al comparar multíparas frente a primíparas) y 
Tabla 2

Variables asociadas con el uso de cesárea. Resultados de la regresión logística multivariante

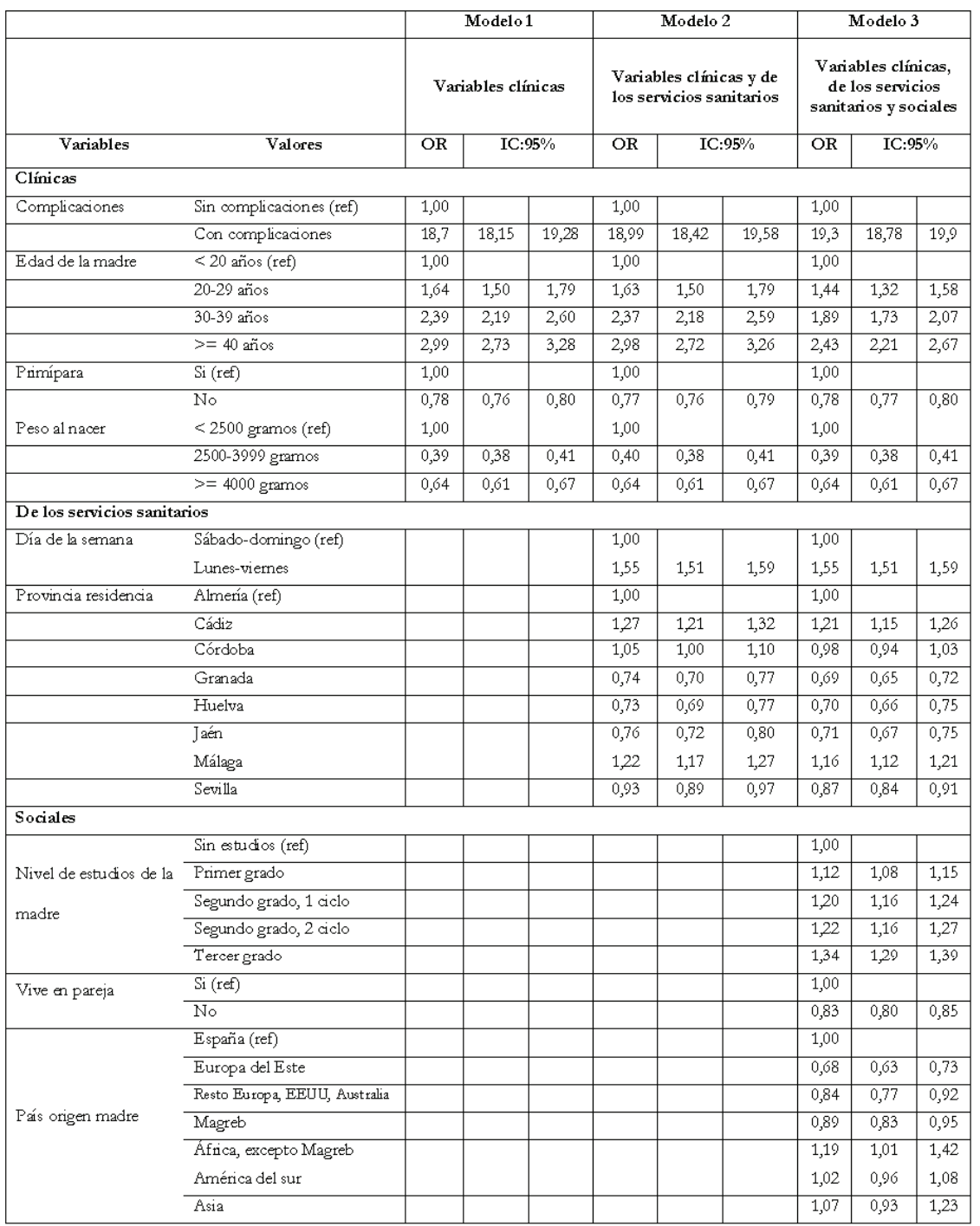


Tabla 3

Porcentajes de cesáreas y razón de partos en hospitales públicos/privados en Andalucía en el año 2007

\begin{tabular}{|l|c|c|c|c|}
\hline Provincias & $\begin{array}{c}\text { \% Cesáreas en } \\
\text { hospitales } \\
\text { públicos }\end{array}$ & $\begin{array}{c}\text { \% Cesáreas en } \\
\text { hospitales } \\
\text { privados }\end{array}$ & $\begin{array}{c}\% \text { Cesáreas } \\
\text { en el total de } \\
\text { hospitales }\end{array}$ & $\begin{array}{c}\text { Razón de partos } \\
\text { en hospitales } \\
\text { públicos/privados }\end{array}$ \\
\hline Almería & 20,6 & 43,2 & 23,5 & 6,8 \\
\hline Cádiz & 24,6 & 45,8 & 29,1 & 3,7 \\
\hline Córdoba & 20,6 & 33,8 & 23,4 & 3,8 \\
\hline Granada & 18,3 & 40,3 & 20,4 & 9,6 \\
\hline Huelva & 22,3 & 27,3 & 22,4 & 45,1 \\
\hline Jaén & 19,0 & 26,3 & 19,1 & 43,0 \\
\hline Málaga & 24,8 & 38,9 & 27,6 & 4,1 \\
\hline Sevilla & 19,8 & 32,1 & 22,7 & 3,3 \\
\hline Andalucía & 21,5 & 37,4 & 24,2 & 4,9 \\
\hline
\end{tabular}

peso al nacer (OR ajustada de 0,39 al comparar el normopeso frente al bajo peso) (tabla 2). Se desestimaron las variables sexo del recién nacido (por presentar una OR muy próxima a la unidad) y tiempo de gestación (por multicolinealidad con el peso del recién nacido).

$\mathrm{Al}$ introducir en el segundo modelo multivariante las variables relacionadas con el funcionamiento de los servicios sanitarios, el día de la semana influyó en la realización de cesáreas (OR de 1,55 al comparar el uso de cesárea de lunes a viernes con los fines de semana), independientemente de la existencia de complicaciones y del resto de las variables clínicas. La asociación del día de la semana con la práctica de cesáreas se mantuvo sin cambios cuando se ajustó por las variables sociales. Las provincias de Cádiz y Málaga tuvieron el uso más alto de cesárea (OR de 1,27 y 1,22 respectivamente al comparar con Almería), si bien esta diferencia disminuyó cuando se contemplaron las variables sociales (tabla 2).

En el tercer modelo multivariante, en el que se añadieron las variables sociales, se mantuvo la asociación entre nivel educativo y riesgo de cesárea que ya se había visto en el análisis descriptivo, con una OR (ajustada por variables clínicas y del sistema sanitario) de 1,34 al comparar las mujeres con estudios de tercer grado respecto a las que no tenían estudios. Las mujeres que no vivían en pareja tuvieron menor uso de cesáreas que las mujeres acompañadas (OR de 0,83). En cuanto al país de origen, las únicas mujeres con mayor uso de cesáreas que las españolas de forma estadísticamente significativa fueron las africanas (excluidas las procedentes del Magreb), con una OR ajustada de 1,19. Por el contrario, el uso de las cesáreas fue menor (al comparar con las españolas) en las mujeres de Europa del Este (OR ajustada de 0,68 ), Magreb (OR ajustada de 0,89), y las del resto de Europa, América del Norte y Australia (OR ajustada de 0,84) (tabla 2).

En la tabla 3 se presentan las cesáreas realizadas en los hospitales públicos: 17.926 $(21,5 \%)$ cesáreas en 83.490 nacimientos y privados: $6.385(37,4 \%)$ cesáreas en 17.072 nacimientos, para el total de Andalucía y por provincias. En las dos provincias asociadas a mayor riesgo de cesáreas en el análisis multivariante, la razón entre nacimientos en centros públicos y privados fue de 3,7 (Cádiz) y 4,1 (Málaga). Asimismo, los porcentajes de cesáreas en las clínicas privadas de estas dos 
provincias son los más elevados de Andalucía, $45,8 \%$ en Cádiz ( 1.365 cesáreas en 2.980 nacimientos) y $38,9 \%$ en Málaga (1.520 cesáreas en 3.904 nacimientos). Dos de las provincias con menos cesáreas (Huelva y Jaén) son las que menos partos en clínicas privadas tuvieron (razón entre nacimientos en centros públicos y privados de 45,1 en Huelva y 43,0 en Jaén).

\section{DISCUSIÓN}

La variable que presenta una asociación de mayor magnitud con la práctica de la cesárea en Andalucía es de índole clínico: la existencia de complicaciones en el nacimiento. No obstante, los resultados también sugieren la existencia de una sobreutilización de este procedimiento quirúrgico en todas las clases sociales, con mayor incidencia en las mujeres de mayor nivel educativo. Asimismo, la influencia de determinados factores relacionados con el funcionamiento del sistema sanitario no es desdeñable, con diferencias interprovinciales que podrían estar reflejando distintos patrones de uso de la medicina privada y menor frecuencia de cesáreas en los nacimientos que ocurren en fines de semana.

Las características de los datos y métodos utilizados están íntimamente relacionadas con los resultados. En este sentido, cabe destacar en primer lugar que este trabajo tiene la ventaja de garantizar la exhaustividad poblacional, al contar con todos los nacimientos acontecidos en Andalucía en el período estudiado (dado que el registro de nacimientos es una obligación legal). Asimismo, los cambios introducidos en el boletín estadístico de partos en el año 2007 han permitido contar con una variable social de especial interés para aproximarse a la clase social: el nivel educativo de la madre.

Sin embargo, a la hora de interpretar los resultados, es fundamental no perder de vista las limitaciones derivadas del uso de una fuente de datos que no ha sido creada para fines de investigación (MNP) y que se ha analizado de forma transversal. Por una parte, no se cuenta con información que sería fundamental para estimar la necesidad clínica de una cesárea, como son los antecedentes de problemas de salud materno-fetales, el modo de presentación fetal $\mathrm{u}$ otros factores. Estas carencias son comunes a otras fuentes de datos y han sido reconocidas por otros autores ${ }^{11}$. A ellas hay que añadir las posibles limitaciones relacionadas con la calidad de los datos de la fuente utilizada ${ }^{12}$. Así, el boletín estadístico de partos es cumplimentado por los padres, y son ellos quienes valoran si el nacimiento ha sido o no complicado. Esta variable (existencia de complicaciones) es muy inespecífica y puede referirse a situaciones distintas. Además, podría estar sujeta a un sesgo de mala clasificación retrospectivo que estaría sobrevalorando la magnitud de la asociación. Por último, el MNP no aporta un dato clave para este estudio, la titularidad de la maternidad (pública o privada), por lo que sólo ha podido hacerse una aproximación al tema a través de otra fuente de datos.

Aún matizando los hallazgos por estas limitaciones, cabe destacar la importante asociación entre las variables con significado clínico y el uso de la cesárea, asociación que se mantiene tras ajustar por variables sociales y otras. Esto es especialmente visible en el caso de la variable "complicaciones", con una elevada OR, hecho que coincide con los resultados de otros estudios que llegan a un nivel mayor de detalle sobre el posible tipo de complicaciones ${ }^{3}$. El gradiente en la frecuencia de cesáreas según edad de la madre y peso del recién nacido coincide también con lo encontrado en otros estudios ${ }^{3,11}$.

En el planteamiento inicial de este estudio, el objetivo principal fue explorar la relación entre el nivel educativo de la madre (como aproximación a la clase social) y el uso de la cesárea. Como está descrito en otros trabajos $^{7,8}$, aquí también se encontró un gradiente por clase social, siendo más frecuente su uso a mayor nivel educativo. Este gradiente 
podría interpretarse desde distintos puntos de vista. Por una parte, podría estar traduciendo una desigualdad de orden social (menor acceso a una intervención necesaria en las clases más desfavorecidas). Sin embargo, la hipótesis de la desigualdad no parece tan acertada si se tiene en cuenta que la tasa de cesáreas en las mujeres de menor nivel educativo es superior a los estándares recomendados en ocho puntos porcentuales ${ }^{9}$. En cualquier caso, la información clínica disponible no permite valorar si existe subutilización en los casos en que no se ha realizado cesárea. Por otra parte, llama la atención que las tasas de cesáreas sean mucho más altas que los estándares de la Organización Mundial de la Salud en todos los niveles educativos y procedencias geográficas. Si bien estos estándares se basaron en el consenso de expertos y no se han modificado desde su publicación en 1985, podría interpretarse que existe un problema de sobreutilización de este procedimiento quirúrgico y enmarcarse dentro de un fenómeno más global de medicalización del nacimiento, que sería más acusado en las clases sociales más altas. Hay otros ejemplos documentados de que la medicalización no ocurre por igual en todas las clases sociales, sino que es mayor en las clases más altas, como se ha puesto de manifiesto en la sobreutilización de las revisiones ginecológicas preventivas en mujeres de mayor nivel educativo $^{13}$.

La relación entre cesárea y estrato social, como muestra un estudio reciente realizado en Barcelona ${ }^{8}$ y las estadísticas de centros sanitarios, está sobre todo mediada por la mayor utilización de los establecimientos privados por las mujeres con mayor nivel educativo. En Andalucía, la frecuencia de cesárea en centros privados casi duplicó la de centros del sistema sanitario público. Al no disponer de información sobre el tipo de centro sanitario en el MNP no se ha podido comprobar si las diferencias por clase social se explican en su totalidad o sólo en parte por este factor.
La clase social se podría relacionar también con el grado de participación de las mujeres en las decisiones sobre el parto. Se ha afirmado que el incremento del uso de la cesárea responde a una demanda de las mujeres ${ }^{14}$, si bien algunas autoras han propuesto que sería más acertado investigar cómo influye la conveniencia de los médicos, basándose en que muchos estudios documentan que la mayoría de las mujeres prefieren a priori el parto vaginal, al tiempo que sugieren que las preferencias de los profesionales juegan un papel clave ${ }^{15}$.

Llama la atención que se siga encontrando el mayor uso de cesáreas en las madres africanas aún después de ajustar por el nivel educativo de la madre y las variables clínicas. Posiblemente este hallazgo no responda a la interpretación que se ha formulado antes para el conjunto de la población, sino que esté reflejando problemas de salud reproductiva relacionados con los controles durante el embarazo. En el otro extremo, el hallazgo de la menor tasa de cesáreas en las mujeres de países del este de Europa concuerda con lo encontrado en otro estudio español ${ }^{16}$ y lo documentado en esta zona geográfica, ya identificada como una de las de menor uso de cesáreas en el mundo ${ }^{17}$.

Por último, merece la pena destacar que del análisis realizado se deriva la existencia de un grado importante de discrecionalidad en la utilización de la cesárea. Dadas las diferencias según día de la semana, titularidad del hospital y provincia, aspectos todos ellos relacionados con los servicios sanitarios. Estos resultados son consistentes con la literatura científica que pone de manifiesto la variabilidad en el uso de la cesárea entre hospitales de similar complejidad y áreas geográficas vecinas ${ }^{6,11,18}$.

A la luz de los hallazgos del presente estudio se pueden sugerir algunas líneas de acción e investigación. Por una parte, parece claro que existe una sobreutilización generalizada de la cesárea al menos en parte asocia- 
da a la práctica en centros privados. Ante esta situación se puede y debe actuar tanto desde la Administración Sanitaria como desde las instituciones y asociaciones profesionales, sobre todo las relacionadas con la obstetricia y matronería. Es fundamental el establecimiento de guías de práctica apropiada y la monitorización de su cumplimiento, así como una revisión de los sistemas de pago por nacimiento a los hospitales privados, sobre todo los que son concertados desde la Administración Sanitaria pública. Asimismo, es clave la transparencia en la información a la población en general y a las mujeres embarazadas en particular. Sería importante que conocieran que un nacimiento no complicado tiene más probabilidad de acabar en cesárea en los centros privados y tener información detallada de sus riesgos. Finalmente, es necesario profundizar en posteriores investigaciones sobre el uso de la cesárea en el colectivo de mujeres inmigrantes, especialmente en las mujeres africanas.

\section{BIBLIOGRAFÍA}

1.Porreco RP, Thorp JA. The cesarean birth epidemics: Trends, causes, and solutions. Am J Obstet Gynecol. 1996;175:369-74.

2.Martínez-Frías ML, Bermejo E, Rodríguez-Pinilla E, Dequino G, Grupo Periférico del ECEMC. Evolución secular y por autonomías de la frecuencia de tratamientos de fertilidad, partos múltiples y cesáreas en España. Med Clin (Barc). 2005;124:132-9.

3.Librero J, Peiró S, Márquez-Calderón S. Inter-hospital variations in caesarean sections. A risk adjusted comparison in the Valencia public hospitals. J Epidemiol Community Health. 2000;54:631-6.

4.Villalbí JR, Navarro A, Plaséncia A. Variabilidad en la práctica de cesáreas. Gac Sanit. 1995;9:140-1.

5.Béhague DP, Victora CG, Barros FC. Consumer demand for caesarean sections in Brazil: informed decision making, patient choice, or social inequality? A population based birth cohort study linking ethnographic and epidemiological methods. BMJ. 2002;324:942-7.

6.Minkoff H, Powderly KR, Chervenak F, McCullough LB. Ethical dimensions of elective primary caesarean delivery. Obstet Gynecol. 2004;103:387-92.
7.Fontoura Freitas P, Drachler ML, Leite JC, Marshall $\mathrm{T}$. Inequalities in caesarean delivery rates by ethnicity and hospital accessibility in Brazil. Int J Gynecol Obstet. $2009 ; 107: 198-201$

8.Salvador J, Cano-Serral G, Rodríguez-Sanz M, Lladonosa A, Borrell $\mathrm{C}$. Inequalities in caesarean section: influence of the type of maternity care and social class in an area with a national health system. J Epidemiol Community Health. 2009;63:259-61.

9. World Health Organization. Appropriate technology for birth. Lancet. 1985;2:436-7.

10.Grupo de Trabajo de la Sociedad Española de Epidemiología. La medición de la clase social en ciencias de la salud. Barcelona: SG Editores, 1995.

11.Bragg F, Cromwell DA, Edozien LC, Gurol-Urganci I, Mahmood TA, Templeton A, et al. Variation in rates of caesarean section among English NHS trusts after accounting for maternal and clinical risk: cross sectional study. B M J . 2010;341: c 5065 doi:10.1136/bmj.c5065.

12.Río I, Castelló A, Jané M, Prats R, Barona C, Más R, et al. Calidad de los datos utilizados para el cálculo de indicadores de salud reproductiva y perinatal en población autóctona e inmigrante. Gac Sanit. 2010;24:172-7.

13.Márquez Calderón S, Villegas Portero R, Aguado Romeo MJ. La utilización de los servicios sanitarios en Andalucía: ¿perpetuación o corrección de las desigualdades? En: Escolar Pujolar A. Primer Informe sobre Desigualdades y Salud en Andalucía. Puerto Real: Asociación para la Defensa de la Sanidad Pública de Andalucía, 2008. Páginas: 145-66.

14.NIH State-of-the-Science Conference Statement on caesarean delivery on maternal request. NIH Consens State Sci Statements. 2006;23:1-29.

15.Bettiol H, Barbieri MA, Moura da Silva AA, Rona RJ. Consumer demand for caesarean sections in Brazil. Demand is affected by mothers' perception of good health care (letter). BMJ.2002;325:336.

16.Río I, Castelló A, Barona C, Jané M, Más R, Rebagliato $\mathrm{M}$, et al. Caesarean section rates in immigrant and native women in Spain: the importance of geographical origin and type of hospital for delivery. Eur J Public Health. 2010;20:524-9.

17.Betrán AP, Merialdi M, Lauer JA, Bing-Shun W, Thomas J, Van Look P, et al. Rates of caesarean section: analysis of global, regional and national estimates. Paediatr Perinat Epidemiol. 2007;21:98-113. 
18.Aelvoet W, Windey F, Molenberghs G, Verstraelen H, Van Reempts P, Foidart JM. Screening for inter-hospital differences in caesarean section rates in low-risk deliveries using administrative data: An initiative to improve the quality of care. BMC Health Serv Res. 2008; 8:3 doi:10.1186/1472-6963-8-3. 